\title{
Clinical characteristics and outcomes of 17 cases of inflammatory myofibroblastic tumor at a University Hospital in China
}

\author{
WEI SONG ${ }^{1}$ and YAN ZHU ${ }^{2}$ \\ Departments of ${ }^{1}$ Pulmonary and Critical Care Medicine, and ${ }^{2}$ Pathology, \\ The First Affiliated Hospital of Nanjing Medical University, Nanjing, Jiangsu 210029, P.R. China
}

Received April 13, 2020; Accepted September 28, 2020

DOI: $10.3892 / \mathrm{ol} .2020 .12312$

\begin{abstract}
The aim of the present study was to explore the clinical and pathological characteristics, diagnosis, and treatment of inflammatory myofibroblastic tumor (IMT). A total of 17 patients with IMT diagnosed between July 2010 and February 2020 were included in the present study, and the clinical characteristics, pathological features, treatment and prognosis were analyzed retrospectively. The cohort consisted of 17 participants, including 12 men and 5 women, with a mean age of 34.76 years. The most common locations of tumors were the bronchi and the lungs ( 9 cases, including 1 case involving the mediastinum), followed by the colon and bladder ( 2 cases each), and the omentum majus, mesocolon, stomach and peritoneal cavity (1 case each). Immunohistochemical staining demonstrated that the tumor cells exhibited positive staining for anaplastic lymphoma kinase p80 (13/17), smooth muscle actin $(12 / 17)$, cytokeratin pan $(6 / 17)$, vimentin $(5 / 17)$ and desmin (4/17). The follow-up time was 18-114 months. A patient with epithelial inflammatory myofibroblast sarcoma (EIMS) succumbed to the disease, 1 case was lost to followup, 2 cases relapsed and the other 13 cases were considered cured. IMTs may be malignant or low-grade. EIMS is a rare and invasive variant of IMT. The clinical and imaging manifestations are often unique and vary among individuals. Once confirmed by pathology, radical surgery should be the first choice of treatment.
\end{abstract}

\section{Introduction}

Inflammatory myofibroblastic tumor (IMT) is a rare type of mesenchymal tumor with malignant potential that is frequently observed in patients who are $<16$ years old, and is

Correspondence to: Dr Yan Zhu, Department of Pathology, The First Affiliated Hospital of Nanjing Medical University, 300 Guangzhou Road, Nanjing, Jiangsu 210029, P.R. China E-mail: zyfz1031@163.com

Key words: inflammatory myofibroblastic tumor, immunohistochemistry, epithelial inflammatory myofibroblast sarcoma, anaplastic lymphoma kinase, treatment rarely observed in adults (1). The World Health Organization (WHO) 2020 definition of IMT suggests that it is a distinctive, rarely metastasizing neoplasm composed of myofibroblastic and fibroblastic spindle cells accompanied by an inflammatory infiltrate of plasma cells, lymphocytes and/or eosinophils (2). IMT exhibits a predilection for the visceral soft tissues, most commonly observed in the lungs, but can develop in any part of the body, including the peritoneum, colon, liver, bladder, breast or nasal cavity (3). IMT arising from somatic soft tissues or extremities has been reported in isolated case reports $(4,5)$. IMTs are genetically heterogeneous (2). Clonal rearrangement of the anaplastic lymphoma kinase (ALK) gene on the short arm of chromosome 2 at 2p23 with fusion of the 3 ' kinase region of the ALK gene with various partners, including tropomyosin 3, tropomyosin 4, clathrin heavy chain and RAN binding protein 2 (RANBP2), has been identified in $50-70 \%$ of IMTs $(2,3)$. Due to the low incidence, particularly in adults, and lack of specificity in clinical and imaging manifestations, IMTs are often misdiagnosed (6). Epithelial inflammatory myofibroblast sarcoma (EIMS), a rare malignant variant of IMT, requires aggressive clinical measures, since patients with EIMS often exhibit rapid local recurrence, and the disease is associated with a high mortality rate (6). At present, only isolated case reports of EIMS exist due to its rarity (7-10).

The present study retrospectively analyzed the clinical features, treatment and prognosis of 17 cases of pathologically diagnosed IMT in patients $\geq 16$ years old who were diagnosed between 2010 and 2020, in order to assist clinicians in improving the recognition and diagnosis of the disease.

\section{Materials and methods}

Study population. IMT tends to occur primarily in patients $<16$ years old, but rarely in those patients $\geq 16$ years old (1). Therefore, a list of all IMT cases with an age $\geq 16$ years confirmed by pathology at The First Affiliated Hospital of Nanjing Medical University (Nanjing, China) was compiled. It was not possible to select younger patients, since the Obstetrics, Gynecology and Paediatrics Departments are located at the Women and Children's Branch Hospital (Nanjing, China).

Data were collected from 17 patients with IMT (age, $\geq 16$ years) diagnosed between July 2010 and February 2020. The diagnosis was confirmed by pathological analysis following surgery, respiratory interventional techniques or 
needle biopsy. All pathological sections were reassessed and the diagnosis was confirmed by two or three pathologists. The present retrospective analysis was approved by the Institutional Review Board of the First Affiliated Hospital of Nanjing Medical University and written informed consent was provided by subjects (adult patients) or next-of-kin (patients $<18$ years old) for inclusion.

Thoracic or abdominal CT or MRI scans were performed based on the location of the primary tumor in all patients and a number of patients underwent a positron emission tomography (PET-CT) scan or a form of endoscopy, including bronchoscopy, gastroenterological endoscopy or cystoscopy, prior to surgery. The present study retrospectively collected the main demographic characteristics and clinical data, including sex, age, presenting symptoms, blood examinations, location, size of tumors, radiological features, endoscopic characteristics and surgical methods, from the medical records. The patients were followed up every 6 months until February 2020 through outpatient visits and/or telephone contact.

Pathological review. The present study reviewed all pathological sections to ensure they matched the criteria of the 5th edition of the WHO classification of tumors of soft tissue and bone (2). Pathologists evaluated the pathological sections for cell morphology, nuclear atypia, vascular invasion and inflammatory components. Immunohistochemistry was performed on a fully automated VENTANA Benchmark XT Stainer (Ventana Medical Systems, Inc.; Roche Diagnostics). The specimens were fixed in $10 \%$ neutral formaldehyde solution at room temperature for $24 \mathrm{~h}$ and embedded into paraffin. The slides (3- $\mu \mathrm{m}$-thick) were heated at $65^{\circ} \mathrm{C}$ for $2 \mathrm{~h}$. Deparaffinization, rehydration and antigen retrieval were automatically completed. All the procedures were performed according to the manufacturer's protocol. The slides were incubated overnight at $4^{\circ} \mathrm{C}$ with pre-diluted (ready-to-use) primary antibodies (Fuzhou Maixin Biotech Co., Ltd.; Table I) and then incubated with goat anti-mouse/rabbit IgG HRP-conjugated polymer secondary antibodies (cat. no. KIT-5230; Fuzhou Maixin Biotech Co., Ltd.) for 20-30 min at room temperature. DAB+ chromogen (Fuzhou Maixin Biotech Co., Ltd.) was used to produce dark brown color. Hematoxylin was used as a counter stain for $5 \mathrm{~min}$ at room temperature. Sections were observed under a light microscope (magnification, x20). Multitumor tissue blocks (including tissues of thyroid, lung, small intestine, liver, tonsil, lymph nodes, prostate, fetal membranes, leiomyoma and lung adenocarcinoma) were used as the positive and negative controls for each immunohistochemical stain analyzed.

Immunohistochemical staining was performed on all samples to confirm the pathological phenotype of IMT, and to differentiate among diseases, such as inflammatory pseudotumours (IPTs), sarcoma, cancer, gastrointestinal stromal tumor, dendritic cell neoplasms or vascular tumors.

Fluorescence in situ hybridization (FISH). Dual color break-apart commercial probes of the ALK kit (Abbott Pharmaceutical Co., Ltd.; cat. no. 06N38) were used for FISH assays. Tissues were fixed in $10 \%$ neutral formaldehyde solution for $24 \mathrm{~h}$ at room temperature, embedded into paraffin and cut into $3-\mu \mathrm{m}$-thick slides. Subsequently, the slides were deparaffinized in xylene for two times (15 min each), and dehy- drated in $95 \%$ ethanol (two times, 5 min each), $80 \%$ ethanol ( $2 \mathrm{~min}), 70 \%$ ethanol (2 min) and double-distilled $\mathrm{H}_{2} \mathrm{O}$ (2 min). The slides were then treated with protease $\mathrm{K}[0.1 \mathrm{mg} / \mathrm{ml}$ in 2X SSC buffer (0.3 M NaCl, $0.03 \mathrm{M}$ sodium citrate, $\mathrm{pH} 7.2)]$ for $10 \mathrm{~min}$ at $37^{\circ} \mathrm{C}$. The slides were washed with $2 \mathrm{X} \mathrm{SSC}$ buffer for two times ( $5 \mathrm{~min}$ ), post-fixed with $100 \%$ ethanol for $2 \mathrm{~min}$ and air-dried at room temperature. For hybridization, $10 \mu 1$ probe dissolved in hybridization buffer $(10 \%$ dextran sulfate, 2X SSC buffer, 50\% deionized formamide) was added to the tissues on slides, which were placed in a Hybrite system (Abbott Pharmaceutical Co., Ltd.) for $5 \mathrm{~min}$ at $83^{\circ} \mathrm{C}$ and then $42^{\circ} \mathrm{C}$ for $4 \mathrm{~h}$. After hybridization, the slides were washed with $0.3 \% \mathrm{NP}-40 / 0.4 \mathrm{X}$ SSC buffer for two times (2 min) at room temperature to remove excess probes. Subsequently, the slides were washed with $70 \%$ ethanol for 3 min and air-dried at room temperature. Finally, $15 \mu \mathrm{l}$ DAPI was added to slides for $5 \mathrm{~min}$ at room temperature to stain the nucleus and mounted. The slides were observed using a fluorescence microscope (DP70; Olympus Corporation). For ALK rearrangement, the nuclei contained broken-apart signals, and for no ALK rearrangement, the specimens contained a set of immediately adjacent or fused orange/green signals.

Statistical analysis. All statistical analyses were performed using the software SPSS 25.0 (IBM Corp.). The data were presented as the mean \pm SD. A Fisher's exact test was used to analyze the data. $\mathrm{P}<0.05$ was considered to indicate a statistically significant difference.

\section{Results}

Patient characteristics. A total of 17 participants, including 12 male and 5 female patients, with a mean \pm SD age of $34.76 \pm 13.79$ years (range, 16-56 years) were included in the present study. The most common locations of occurrence were the bronchus and lungs (9 cases, 52.94\%), one of which was a patient with multiple sites of involvement, including the mediastinum. The other sites of involvement included colon and bladder ( 2 cases each; 11.76\%), and omentum majus, mesocolon, stomach and peritoneum (1 case each; 5.88\%). Four cases had a history of smoking or alcoholism, and another 4 cases had a history of trauma or surgery (data not shown).

A total of 5 cases $(29.41 \%)$ were discovered by physical examination, regional pain in the lesions accounted for 4 cases $(23.53 \%)$, and hemoptysis, melena or hematuria were the main symptoms in 4 cases $(23.53 \%)$, followed by tumor masses and fever ( 2 cases each; 11.76\%). The time interval between symptom onset and diagnosis was between 1 week and 8 years (data not shown). A 16-year-old patient with greater omentum IMT presented with fever, anemia and thrombocytosis before surgery; however, the symptoms gradually disappeared after surgery. The patient with EIMS (case 13) presented with 1 week of abdominal distention, and 3 days of oliguria and diarrhea. The abdominal CT scan revealed abdominal and pelvic effusion, a thickened peritoneum, and an unclear structure in the mid-abdomen. Blood examination revealed that the white blood cell and platelet counts were elevated.

Radiological features before treatment. The most common radiological manifestations were masses of different sizes 

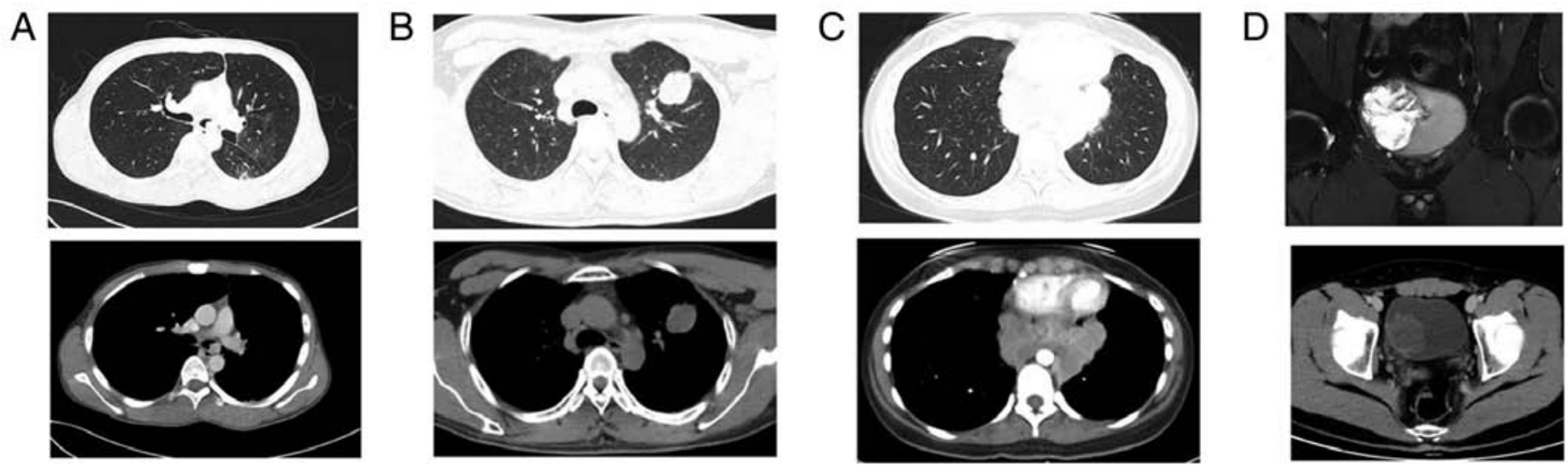

Figure 1. Radiological analysis revealing tumors of different sizes and in different locations. CT scan images of (A) tumor in the left main bronchus, (B) tumor in the left upper lobe with pleural indentation and (C) tumor in the posterior mediastinum with evidence of invasion into the esophagus. Upper panels show the lung window, while lower panels show the mediastinal window. (D) MRI image of bladder tumor. Upper panel is a T2-weighted image, while the lower panel is a T1-weighted image.

Table I. Primary antibodies used for immunohistochemistry purchased from Fuzhou Maixin Biotech Co., Ltd.

\begin{tabular}{lll}
\hline Antibody & Catalogue. number & $\begin{array}{c}\text { Poly/monoclonal } \\
\text { clone number }\end{array}$ \\
\hline ALK & MAB-0281 & 5A4 \\
SMA & Kit-0006 & 1A4 \\
Vim & MAB-0735 & MX034 \\
Des & MAB-0766 & MX046 \\
Ki-67 & MAB-0672 & MX006 \\
CKpan & Kit-0009 & AE1/AE3 \\
S-100 & Kit-0007 & 4C4.9 \\
CD21 & MAB-0339 & 2G9 \\
CD30 & MAB-0023 & Ber-H2 \\
CD117 & kit-0029 & YR145 \\
CD163 & MAB-0206 & 10D6 \\
CD68 & Kit-0026 & KP1 \\
HMB-45 & MAB-0098 & HMB45 \\
DOG1 & Kit-0035 & SP31
\end{tabular}

ALK, anaplastic lymphoma kinase; SMA, smooth muscle actin; Vim, vimentin; Des, desmin; CK, cytokeratins; CD, cluster of differentiation; HMB, human melanoma black; DOG1, anoctamin-1.

(Fig. 1). The CT or MRI diagnoses before treatment were as follows: 10 patients were diagnosed with malignant tumors, 2 with lymphoma, 3 cases were considered benign lesions, including 2 cases of granulomatous disease and 1 pulmonary sclerosing hemangioma. In addition, 2 cases of peritoneal effusion were separately considered to be stromal tumor and tuberculous peritonitis. Additionally, 3 patients who underwent PET-CT all exhibited ${ }^{18} \mathrm{~F}$-fluorodeoxyglucose uptake, with a diagnosis of malignant tumor with lymph node metastasis in 1 case and granulomatous disease in 2 cases (data not shown).

Treatment and follow-up. Among the 17 patients, 1 patient was diagnosed with EIMS by needle biopsy under the guidance of ultrasound and survived only 3 weeks from symptom onset, and thus did not undergo surgery. The remaining 16 patients underwent tumor resection by surgery or respiratory interventional techniques. Furthermore, 2 cases relapsed, and both of these patients underwent surgery again, and 1 of these patients was administered crizotinib following reoperation, while the other received local interventional therapy following recurrence due to negative ALK expression.

All patients were followed up until February 2020 through outpatient visits and/or phone calls to patients themselves or family members. One patient was lost to follow-up, 1 patient died, 2 local recurrence patients (case 9 and 16) were confirmed to be alive, and the remaining 13 patients were cured. The follow-up time of each patient was $>1$ year. The average follow-up time was 48.93 months (range, 18-114 months).

The clinical characteristics, primary locations, tumor sizes, treatment and prognosis of the 17 patients are summarized in Table II.

Clinical characteristics by age group and location of the lesion. In total, 9 patients were $<40$ years old and had a mean age of onset of 23.11 years. The patients who exhibited recurrence and the patient with EIMS were all in this group. The lesion locations were the lungs/mediastinum (4 cases), omentum majus/ mesocolon/peritoneum (3 cases), and colon and bladder (1 case each). The other 8 patients were $>40$ years old, the mean age of onset was 47.88 years, all patients underwent surgery and were cured, and the lesion locations were the lungs (5 cases), and stomach, bladder and colon (1 case each; Table III).

In total, 9 patients exhibited a primary tumor located in the lungs/mediastinum (52.94\%), and the remaining 8 patients exhibited tumors located in the abdomen. Cases of IMT in the oral and maxillofacial region, breast or nervous system were not observed in the present study.

Pathological characteristics. The resected tumor sizes ranged between 0.7 and $15 \mathrm{~cm}$ in the largest dimension, with a mean size of $4.59 \mathrm{~cm}$. Gross examination revealed that the tumors exhibited soft to firm variegated appearance with grey white areas, and parts of the mass presenting a polypoidlike appearance protruding into the bronchus, bladder or 


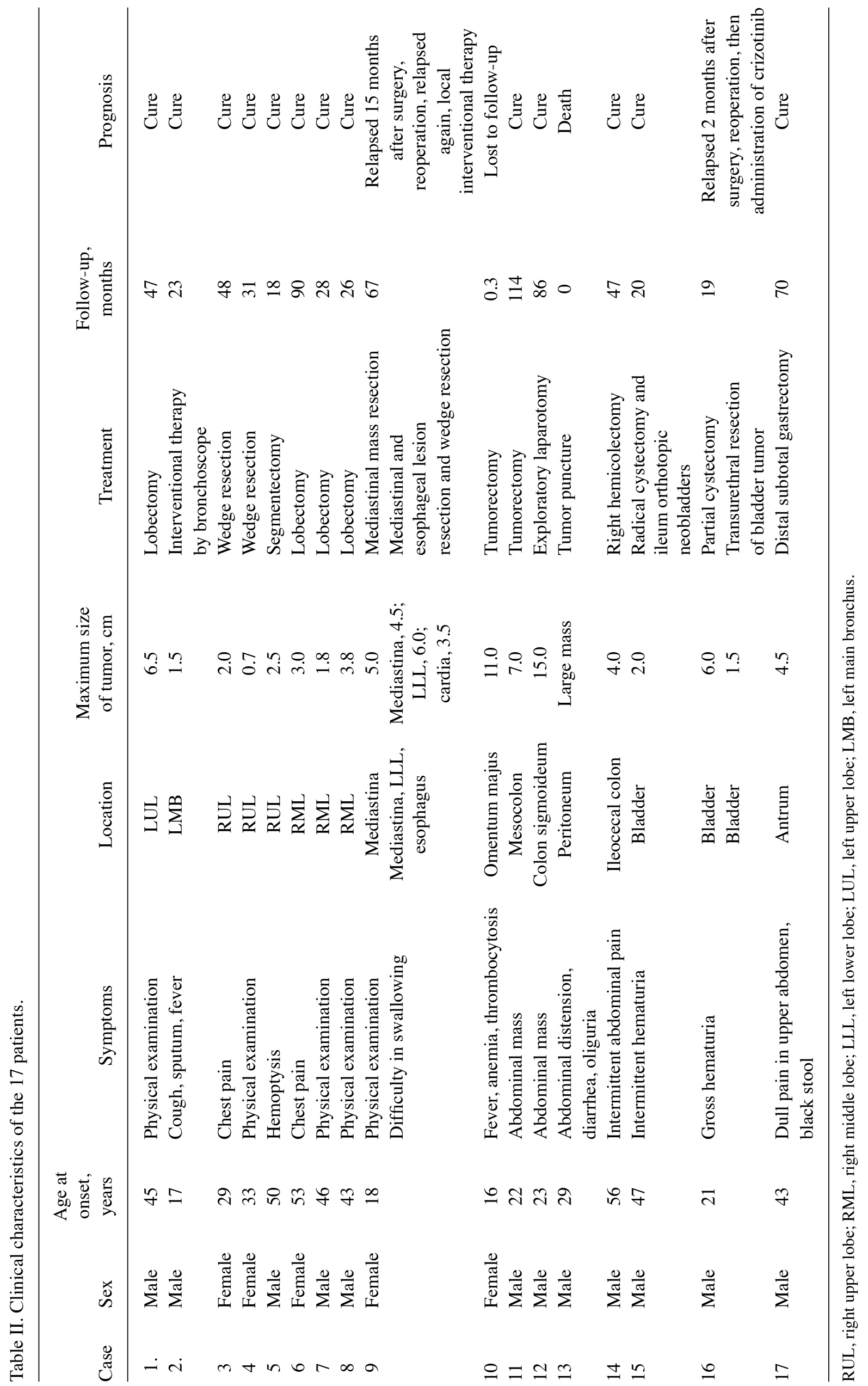


Table III. Clinical characteristics based on age and intra/extra-thoracic location.

\begin{tabular}{|c|c|c|c|c|c|c|}
\hline \multirow[b]{2}{*}{ Clinical characteristics } & \multicolumn{3}{|c|}{ Age } & \multicolumn{3}{|c|}{ Lesion location } \\
\hline & $\leq 40$ years, $n$ & $>40$ years, $n$ & P-value & Intra-thoracic, $\mathrm{n}$ & Extra-thoracic, $\mathrm{n}$ & P-value \\
\hline Sex & & & 0.29 & & & 0.29 \\
\hline Male & 5 & 7 & & 5 & 7 & \\
\hline Female & 4 & 1 & & 4 & 1 & \\
\hline Lesion location & & & 0.64 & & & \\
\hline Intra-thoracic & 4 & 5 & & & & \\
\hline Extra-thoracic & 5 & 3 & & & & \\
\hline Neoplasm invasiveness & & & 0.64 & & & 0.35 \\
\hline No & 4 & 5 & & 6 & 3 & \\
\hline Yes & 5 & 3 & & 3 & 5 & \\
\hline Initial treatment & & & $>0.99$ & & & 0.06 \\
\hline MIS or endoscopy & 5 & 6 & & 8 & 3 & \\
\hline Routine surgery & 3 & 2 & & 1 & 4 & \\
\hline Non-surgery & 1 & 0 & & 0 & 1 & \\
\hline Lymph node dissection & & & $<0.01$ & & & $>0.99$ \\
\hline Yes & 1 & 8 & & 5 & 4 & \\
\hline No & 7 & 0 & & 4 & 3 & \\
\hline NA & 1 & 0 & & 0 & 1 & \\
\hline Relapse & & & 0.08 & & & 0.44 \\
\hline Yes & 2 & 0 & & 1 & 1 & \\
\hline No & 5 & 8 & & 8 & 5 & \\
\hline NA & 2 & 0 & & 0 & 2 & \\
\hline
\end{tabular}

MIS, minimally invasive surgery; NA, not applicable.
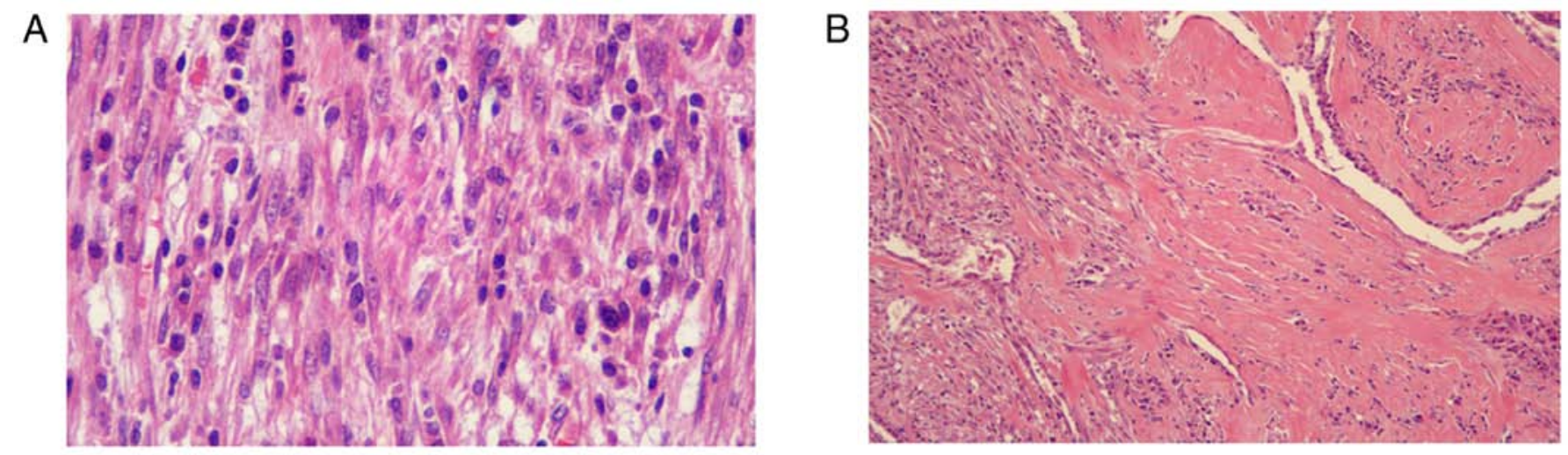

Figure 2. Cytomorphological analysis of an inflammatory myofibroblastic tumor. (A) Long spindle cells contained small nuclei with vesicular chromatin and scattered plasma cells and lymphocytes. Magnification, x400. (B) Pulmonary inflammatory myofibroblastic tumor exhibiting distinct collagenous stroma with scattered plasma cell infiltrate. Magnification, x100.

colon (data not shown). Microscopically, the plump spindle myofibroblasts and fibroblast-like tumor cells were arranged loosely or densely with a fascicular or storiform architecture in a loose edematous or collagenous stroma, with a prominent presence of acute and chronic inflammatory cell infiltration, consisting primarily of plasma cells and lymphocytes (Fig. 2). According to immunohistochemistry (Table IV), the positive rate of ALKp80 expression (Fig. 3A) was the highest (13/17), followed by SMA (12/17; Fig. 3B), CKp (6/17; Fig. 3C), Vim (5/17; Fig. 3D) and Des (4/17). Identification of ALK gene rearrangement by FISH was performed in 5 patients, and only 2 demonstrated evidence of ALK rearrangement (Fig. 4). In the patient with EIMS, the Ki-67 index was $10 \%$, and in the two relapsed patients, the Ki-67 index was 5\%+ and 3\% (data not shown); only in 2 patients, the $\mathrm{Ki}-67$ index was $10 \%+$ (Table IV). 
Table IV. Pathological characteristics based on age and intra/extra-thoracic location

\begin{tabular}{|c|c|c|c|c|c|c|}
\hline \multirow[b]{2}{*}{ Pathological characteristics } & \multicolumn{3}{|c|}{ Age } & \multicolumn{3}{|c|}{ Lesion location } \\
\hline & $\leq 40$ years, $n$ & $>40$ years, $n$ & P-value & Intra-thoracic, $\mathrm{n}$ & Extra-thoracic, $n$ & P-value \\
\hline Maximum tumor diameter, $\mathrm{cm}$ & & & 0.13 & & & 0.05 \\
\hline$\leq 5$ & 4 & 7 & & 8 & 3 & \\
\hline$>5$ & 5 & 1 & & 1 & 5 & \\
\hline $\begin{array}{l}\text { ALK rearrangements } \\
\text { by immunohistochemistry }\end{array}$ & & & $>0.99$ & & & 0.58 \\
\hline Positive & 7 & 6 & & 6 & 7 & \\
\hline Negative & 2 & 2 & & 3 & 1 & \\
\hline SMA staining & & & 0.13 & & & $>0.99$ \\
\hline Positive & 8 & 4 & & 6 & 6 & \\
\hline Negative & 1 & 4 & & 3 & 2 & \\
\hline CKp staining & & & $>0.99$ & & & $<0.01$ \\
\hline Positive & 3 & 3 & & 0 & 6 & \\
\hline Negative & 6 & 5 & & 9 & 2 & \\
\hline Vim staining & & & $>0.99$ & & & 0.13 \\
\hline Positive & 3 & 2 & & 1 & 4 & \\
\hline Negative & 6 & 6 & & 8 & 4 & \\
\hline Des staining & & & $>0.99$ & & & 0.29 \\
\hline Positive & 2 & 2 & & 1 & 3 & \\
\hline Negative & 7 & 6 & & 8 & 5 & \\
\hline Ki-67 index & & & 0.21 & & & $>0.99$ \\
\hline$\leq 10 \%$ & 9 & 6 & & 8 & 7 & \\
\hline$>10 \%$ & 0 & 2 & & 1 & 1 & \\
\hline
\end{tabular}

ALK, anaplastic lymphoma kinase; Des, desmin; SMA, smooth muscle actin; CK, cytokeratins; Vim, vimentin.
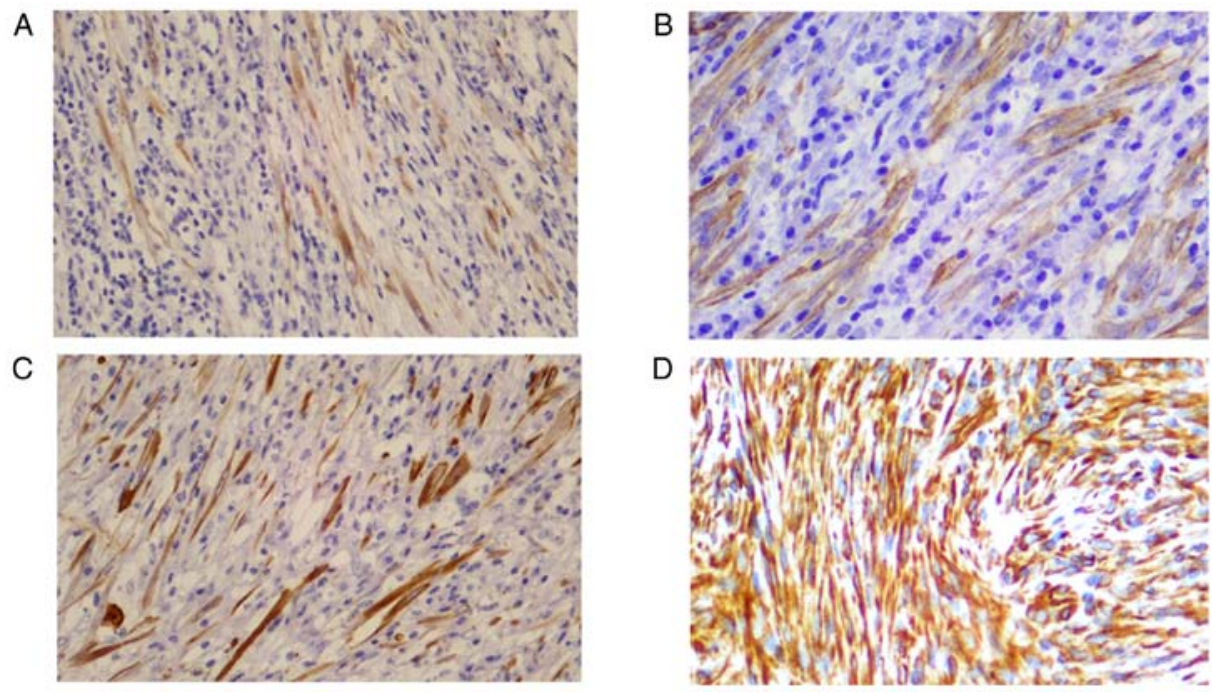

Figure 3. Immunohistochemistry analysis of tissues. (A) Positive staining for anaplastic lymphoma kinase in the cytoplasm of tumor cells. (B) Neoplastic cells positive for smooth muscle actin. (C) Positive staining for cytokeratin pan in certain tumor cells. (D) Vimentin-positive cells. Magnification, x200.

\section{Discussion}

IMTs are rare mesenchymal neoplasms which exhibit low to intermediate malignant potential, and are composed of spindle shaped myofibroblasts often accompanied by the presence of inflammatory cells $(3,11)$. IMTs are referred to by several terms, including IPT, histiocytoma, fibrous histiocytoma, xanthoma, xanthofibroma, xantogranuloma and 


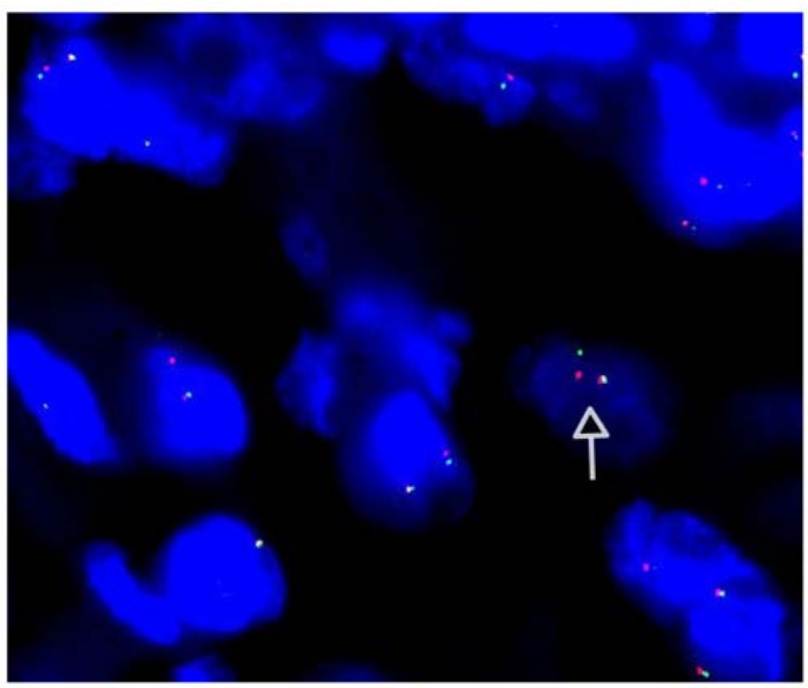

Figure 4. FISH analysis of the ALK locus. FISH revealed the typical splitsignal pattern (arrow), demonstrating the presence of ALK rearrangement. Magnification, x1,000. ALK, anaplastic lymphoma kinase; FISH, fluorescence in situ hybridization.

plasma cell granuloma (1). IPT has been the most widely used term, and IMT has previously been considered to be a type of IPT. At present, some clinicians still use the terms ITP and IMT interchangeably. IPT is an inflammatory reactive mass, which is currently considered to be an IgG4-related disease (12). Although they exhibit certain similarities with regards to morphology, IMT and IPT are different diseases. The therapeutic principles are also different. IMT is treated using surgical excision, whereas IPT can be managed more conservatively (3). Generally, the proliferation and nuclear atypia of spindle cells in IMT are more marked, and ALK-1 staining is positive in some patients (13). Lymphoplasmacytic infiltrate, storiform-fibrosis and obliterative phlebitis (14) are more prominent in IgG4-related IPT, whereas ALK expression is negative $(12,13)$. Next generation sequencing-based RNA fusion assays have been suggested to be a primary method, which may be used to provide a more accurate diagnosis than immunohistochemical platforms and FISH-based assays of ALK (14).

IMT affects patients of a broad age range, but generally presents in patients $<16$ years old (1). The most common anatomical location of IMT is the lung; however, theoretically, any site may be involved, including the mesentery, retroperitoneum, mediastinum, somatic soft tissues, larynx, uterus, bone and central nervous system (3). In the present study, the primary locations were the lungs and the intra-abdominal regions, 1 case presented with a tumor in the mediastinum, and cases in other organs were not detected.

To the best of our knowledge, the etiology and pathogenesis of IMT is unknown. Trauma, surgery, inflammation and infection may contribute to the development of IMT (15). Epstein-Barr virus (16) and human herpesvirus- 8 viral infection (3) are also considered to be associated with IMT; however, additional evidence is required, and a causal relationship has not been established. In the present study, 4 patients had a history of trauma or surgery, but the time span between trauma or surgery and IMT onset was 2-10 years, and the loca- tion of trauma or surgery was not the same as that of IMT. Additionally, 4 patients had a history of smoking, but the IMT locations were not all in bronchus or lung. However, in the patient with a history of alcoholism, the lesion was located in the gastric antrum. Therefore, whether these were associated with the occurrence of IMT was unclear. In addition, 2 patients were assessed for Epstein-Barr encoded RNA and both were negative, suggesting that IMT was not associated with Epstein-Barr virus infection.

Patients usually present with a mass or nonspecific symptoms, which vary according to the location, size and growth pattern of the tumor; additionally, the radiological features are also non-specific (3). In the present study, the diagnoses based on imaging prior to surgery were mostly cancer, stromal tumor or lymphoma. Out of 6 patients, some underwent gastroenterological endoscopy, while others received bronchoscopy or cystoscopy prior to surgery; however, all had negative results. After surgery, immunohistochemistry analysis was required to confirm the pathological diagnosis. A 16-year-old patient with IMT presented with fever, anemia and thrombocytosis before surgery, the large tumor (11x8x8 cm in the greater omentum) required an increased nutrient supply, which resulted in anemia, while the inflammatory stimulation of the tumor caused fever and thrombocytosis. All these parameters gradually returned to normal following surgery. The histological characteristics of IMT include variable spindle cell proliferation in a myxoid to collagenous stroma, with prominent inflammatory infiltrate composed primarily of plasma cells and lymphocytes, with the occasional presence of neutrophils and eosinophils (3). Immunohistochemistry is performed primarily to confirm the immunophenotype of myofibroblasts and to exclude other diseases. ALK rearrangement is relatively common in IMT, while it is negative in leiomyosarcoma, sarcomatoid carcinoma, embryonal rhabdomyosarcoma or reactive myofibroblastic proliferations (17). The present study revealed a $76 \%$ positive rate of ALK staining, in line with previous literature showing that ALK-1 positive staining is observed in $60-89 \%$ of cases (18). ALK-positive patients appear to have a favorable prognosis $(11,15)$. For patients with ALK-positive IMT with unresectable tumors or with advanced stage IMT, ALK inhibition is an effective therapy (19). Watanabe et al (20) reported on a patient with ALK-negative primary pulmonary IMT who achieved remission when treated with macrolide drugsclarithromycin. The Ki-67 index in the patient with EIMS was $10 \%$, and in the two patients with relapse it was $5 \%+$ and $3 \%$, respectively, which was not higher than that of the other patients. A high Ki-67 index may be a risk factor for tumor progression, but was not associated with relapse.

EIMS is a rare variant of IMT, which is clinically distinct from conventional cases in that it follows an aggressive clinical course and has a high mortality rate (6). EIMS predominantly originates intraabdominally, with most cases arising in the omentum and mesentery, and adult males appear to be more susceptible to EIMS (7). There are some common features observed in patients with EIMS, including round-to-epithelioid tumor cells, abundant myxoid stroma with inflammatory infiltrates composed primarily of neutrophils, immuno-positivity for nuclear membrane ALK staining, RANBP2-ALK gene fusion and Des positive staining $(7,8)$. In IMTs, inflammatory infiltrates are composed primarily of 
plasma cells and lymphocytes (3), immuno-positive staining for ALK expression is predominantly observed in the cytoplasm and membrane (9), and SMA expression is more commonly observed than Des (3). Lee et al (9) reported a novel ribosome binding protein 1 (RRBP1)-ALK fusion gene in EIMS, and, to the best of our knowledge, RRBP1-ALK and RANBP2-ALK are the only recurrent oncogenic mechanisms identified to date in EIMS. Although the prognosis of patients with EIMS is poor, there are still a few cases of successful remission with a sustained response to the ALK inhibitor crizotinib (10). Furthermore, programmed cell death 1 ligand 1 expression has been detected in patients with EIMS (7). Therefore, whether immune checkpoint blockade may serve as a novel therapeutic approach for treatment of EIMS should be studied further. In the present study, one patient was diagnosed with peritoneum EIMS, although ALK fusion gene detection was positive, the patient still succumbed to the disease, as the disease was present at an advanced stage at symptom onset and progressed rapidly thereafter.

During the collection of clinical data and rechecking of the pathological data, it was identified that IMTs were occasionally over-diagnosed. Under most circumstances, myofibroblast hyperplasia was diagnosed as IMT. Therefore, one should be wary of diagnosing a middle-aged or elderly patient with IMT. The present study was limited by the small number of cases, and ALK gene rearrangement assessment by FISH was only performed in some patients. A lack of younger cases was another limitation of the present study. Due to this selection bias, the positive rate of ALK-positive staining may be different from that in patients of all ages with IMT. Additional clinical data are required to identify prognostic features which could be used to guide treatment and predict the outcome.

In conclusion, IMT is a low to intermediate-grade tumor which exhibits low potential for malignancy, and has a tendency for local recurrence and a small risk of distant metastasis. The clinical manifestations and imaging findings are usually non-specific. Once the histopathological diagnosis is confirmed, radical surgery is preferred and routine follow-up is necessary.

\section{Acknowledgements}

Not applicable.

\section{Funding}

No funding was received.

\section{Availability of data and materials}

The datasets used and/or analyzed during the current study are available from the corresponding author on reasonable request.

\section{Authors' contributions}

WS conceived the study, collected and analysed the patient data, and was a major contributor in writing the manuscript. YZ designed the study and performed the histological and immunohistochemistry examination. All authors read and approved the final manuscript.

\section{Ethics approval and consent to participate}

The present retrospective analysis was approved by the Institutional Review Board of the First Affiliated Hospital of Nanjing Medical University and written informed consent was provided by the subjects (adult patients)/next-of-kin (patients $<18$ years old) for inclusion.

\section{Patient consent for publication}

Not applicable.

\section{Competing interests}

The authors declare that they have no competing interests.

\section{References}

1. Oztuna F, Pehlivanlar M, Abul Y, Tekinbas C, Ozoran Y and Ozlu T: Adult inflammatory myofibroblastic tumor of the trachea: Case report and literature review. Respir Care 58: e72-e76, 2013.

2. Yamamoto H: WHO Classification of Tumours Editorial Board. Fibroblastic/myofibroblastic Tumors. WHO Classification of Tumors of Soft Tissue and Bone. 5th edition. International Agency for Research on Cancer, Lyon, France, pp 109-112, 2020.

3. Gleason BC and Hornick JL: Inflammatory myofibroblastic tumours: Where are we now? J Clin Pathol 61: 428-437, 2008.

4. Ding R, Li X, Zhu XM, Song QX, Fan QH, Zhang ZH, Gong QX: Inflammatory myofibroblastic tumor arising from soft tissues of extremities harboring a novel CLIP2-ALK fusion. Pathol Int 70: 798-803, 2020

5. Liu H, Lin J, Yang P, Shen H and Yang H: Whether inflammatory myofibroblastic tumor of the thigh relapses after surgical excision? Int J Clin Exp Med 8: 11584-11588, 2015.

6. Mariño-Enríquez A, Wang WL, Roy A, Lopez-Terrada D, Lazar AJ, Fletcher CD, Coffin CM and Hornick JL: Epithelioid inflammatory myofibroblastic sarcoma: An aggressive intraabdominal variant of inflammatory myofibroblastic tumor with nuclear membrane or perinuclear ALK. Am J Surg Pathol 35: 135-144, 2011.

7. Du X, Gao Y, Zhao H, Li B, Xue W and Wang D: Clinicopathological analysis of epithelioid inflammatory myofibroblastic sarcoma. Oncol Lett 15: 9317-9326, 2018.

8. Sarmiento DE, Clevenger JA, Masters GA, Bauer TL and Nam BT: Epithelioid inflammatory myofibroblastic sarcoma: A case report. J Thorac Dis 7: E513-E516, 2015.

9. Lee JC, Li CF, Huang HY, Zhu MJ, Mariño-Enríquez A, Lee CT, Ou WB, Hornick JL and Fletcher JA: ALK oncoproteins in atypical inflammatory myofibroblastic tumours: Novel RRBP1-ALK fusions in epithelioid inflammatory myofibroblastic sarcoma. J Pathol 241: 316-323, 2017.

10. Liu Q, Kan Y, Zhao Y, He H and Kong L: Epithelioid inflammatory myofibroblastic sarcoma treated with ALK inhibitor: A case report and review of literature. Int J Clin Exp Pathol 8: 15328-15332, 2015.

11. Shukla PS and Mittal K: Inflammatory myofibroblastic tumor in female genital tract. Arch Pathol Lab Med 143: 122-129, 2019.

12. Chougule A, Bal A, Das A, Agarwal R, Singh N and Rao KL: A comparative study of inflammatory myofibroblastic tumors and tumefactive IgG4-related inflammatory lesions: The relevance of IgG4 plasma cells. Appl Immunohistochem Mol Morphol 24: 721-728, 2016.

13. Zhu L, Li J, Liu C, Ding W, Lin F, Guo C and Liu L: Pulmonary inflammatory myofibroblastic tumor versus IgG4-related inflammatory pseudotumor: Differential diagnosis based on a case series. J Thorac Dis 9: 598-609, 2017.

14. Taylor MS, Chougule A, MacLeay AR, Kurzawa P, Chebib I, Le L and Deshpande V: Morphologic overlap between inflammatory myofibroblastic tumor and IgG4-related disease: Lessons from next-generation sequencing. Am J Surg Pathol 43: 314-324, 2019.

15. Pecoraro Y, Diso D, Anile M, Russo E, Patella M and Venuta F: Primary inflammatory myofibroblastic tumor of the trachea. Respirol Case Rep 2: 147-149, 2014. 
16. Wang $\mathrm{S}$, Chen $\mathrm{L}$, Cao Z, Mao X, Zhang $\mathrm{L}$ and Wang $\mathrm{B}$ Inflammatory myofibroblastic tumor of the lumbar spinal canal: A Case Report With Literature Review. Medicine (Baltimore) 96: e6488, 2017.

17. Sukov WR, Cheville JC, Carlson AW, Shearer BM,Piatigorsky EJ, Grogg KL, Sebo TJ, Sinnwell JP and Ketterling RP: Utility of ALK-1 protein expression and ALK rearrangements in distinguishing inflammatory myofibroblastic tumor from malignant spindle cell lesions of the urinary bladder. Mod Pathol 20: 592-603, 2007.

18. Cook JR, Dehner LP, Collins MH, Ma Z, Morris SW, Coffin CM and Hill DA: Anaplastic lymphoma kinase (ALK) expression in the inflammatory myofibroblastic tumor: A comparative immunohistochemical study. Am J Surg Pathol 25: 1364-1371, 2001.
19. Mossé YP, Voss SD, Lim MS, Rolland D, Minard CG, Fox E, Adamson P, Wilner K, Blaney SM and Weigel BJ: Targeting ALK with crizotinib in pediatric anaplastic large cell lymphoma and inflammatory myofibroblastic tumor: A children's oncology group study. J Clin Oncol 35: 3215-3221, 2017.

20. Watanabe H, Uruma T, Tazaki G, Tajiri T, Kikuchi R, Itoh M, Aoshiba $\mathrm{K}$ and Nakamura $\mathrm{H}$ : Remission of ALK-negative primary pulmonary inflammatory myofibroblastic tumor on treatment with clarithromycin: A case report and review of the literature. Oncol Lett 11: 1757-1761, 2016.

(i) (8) This work is licensed under a Creative Commons Attribution-NonCommercial-NoDerivatives 4.0 International (CC BY-NC-ND 4.0) License. 